\title{
Variabilidad espacial y temporal de huevos y larvas de Strangomera bentincki y Engraulis ringens, asociados a la desembocadura del río Itata, Chile
}

Variations in space and time of eggs and larvae of Strangomera bentincki and Engraulis ringens associated to the Itata River mouth, Chile

\author{
Samuel Soto-Mendoza ${ }^{1,2}$, Leonardo R. Castro ${ }^{1,2,3}$ y Alejandra Llanos-Rivera ${ }^{1,2,4}$ \\ ${ }^{1}$ Laboratorio de Ecología Pesquera y Oceanografía Larval (LOPEL), Departamento de Oceanografía, Universidad de Concepción, \\ Casilla 160-C, Concepción, Chile. sasoto@udec.cl \\ ${ }^{2}$ Programa de Investigación Marina de Excelencia (PIMEX), Universidad de Concepción, Casilla 160-C, Concepción, Chile \\ ${ }^{3}$ Departamento de Oceanografía y Centro FONDAP-COPAS, Universidad de Concepción, Casilla 160-C, Concepción, Chile \\ ${ }^{4}$ Unidad de Biotecnología, Facultad de Ciencias Naturales y Oceanográficas, Universidad de Concepción, Casilla 160-C, \\ Concepción, Chile
}

\begin{abstract}
This study assesses the spatial and temporal variations in eggs and larval distributions of Engraulis ringens and Strangomera bentincki during their peak and secondary spawning periods, in the zone adjacent to the Itata River mouth, Chile. The information on abundance and distribution of the early life stages of both fish species was obtained from seven oceanographic cruises performed out between 1996 and 2005. The abundance and distribution of eggs and preflexión larvae of both species and, in order to detect potential variations in abundance was analyzed. Our results showed that eggs of both species tended to aggregate at the coast. The spatial extensions of preflexion larvae were broader than that of eggs of both species, along a coast- to- ocean direction. The distribution of the early life stages of both species over the continental shelf off the Itata River mouth was variable inter-annually. These egg and larval abundances showed significant variations among years and the eggs abundance was higher than of larvae. The highest egg and larval abundances occurred at salinities between 32 psu and 34 psu. However, occasionally it was possible to find aggregations in lower salinities (20-24 psu) in the nearby zone of the Itata river mouth. The area contiguous to the river mouth, however, showed low abundance of egg and larvae during the period studied.
\end{abstract}

Key words: Anchoveta, common sardine, eggs, preflexion larvae

\begin{abstract}
Resumen.- El presente estudio analiza la distribución espacial y variabilidad temporal de huevos y larvas de Strangomera bentincki y Engraulis ringens en los periodos de desove principal y secundario, en la zona adyacente a la desembocadura del río Itata, Chile. La información se obtuvo de 7 cruceros oceanográficos entre 1996 a 2005 . Se analizó la abundancia y distribución espacial de huevos y larvas en preflexión para ambas especies y la magnitud de las variaciones en su abundancia. Los resultados mostraron que huevos de ambas especies tienden a concentrarse en la costa. La cobertura espacial de larvas en preflexión es mayor que en huevos, en dirección costa-océano. La distribución de los estadios tempranos de sardina común y anchoveta en la plataforma continental frente a la desembocadura el Río Itata fueron variables inter-anualmente. La variación de abundancia entre años fue mayor para huevos que para larvas en ambas especies siendo mayor en los huevos que en las larvas. Las mayores abundancias de huevos y larvas de anchoveta y sardina común se encontraron en salinidades entre los 32 psu y 34 psu, sin embargo, en ocasiones (año 2005) fue posible observar agrupaciones en salinidades menores ( 20 a 24 psu) en la zona adyacente a la desembocadura del río Itata. El área inmediatamente adyacente a la desembocadura, sin embargo, presentó baj as abundancias de huevos y larvas de estas especies durante los periodos estudiados.
\end{abstract}

Palabras clave: Anchoveta, sardina común, huevos, larvas en preflexión

\section{INTRODUCCIÓN}

La anchoveta (Engraulis ringens) y sardina común (Strangomera bentincki) son especies claves en sistemas de surgencia costera y de gran importancia comercial a lo largo de sus rangos de distribución latitudinal. En la zona centro sur de Chile, ambas especies han producido desembarques que fueron entre las $400.000 \mathrm{y}$ las 1.500 .000 ton en la década de los 90 (Cubillos et al. 2002). La distribución de anchoveta va desde Punta Aguja ( $6^{\circ} \mathrm{S}$, Perú) hasta el sur de Chiloé (42031's, Chile) y la de sardina común desde Valparaíso (3302’s) hasta Puerto Montt 
(412’’S) (Serra 1978, Serra et al. 1979). Mientras tres stocks mayores han sido descritos para la anchoveta (norte de Perú, sur de Perú-Norte de Chile, y centro-sur de Chile), solo un stock ha sido descrito para la sardina común (centro-sur de Chile) (Castillo et al. 1995, Frimodt 1995, Ayon 2001). Ambas especies se encuentran principalmente entre los primeros 30 a $50 \mathrm{~km}$ de la costa y en profundidades de 3 a $80 \mathrm{~m}$ (Serra 1986, Castillo et al. 1995, Frimodt 1995). La anchoveta y sardina común se caracterizan por presentar un desove anual continuo y asociado a zonas costeras someras, observándose un período de desove principal entre los meses de inviernoprimavera (julio-diciembre) para la anchoveta y a fines de invierno (julio-septiembre) para la sardina común (Cubillos \& Arancibia 1993, Arcos et al. 1996). Dentro del área de desove centro-sur de Chile, las mayores abundancias de huevos han sido observadas en cuatro zonas: Punta Nugurne a Constitución (35³0’S), desembocadura del Río Itata a Bahía Concepción (36³0’S), Golfo de Arauco

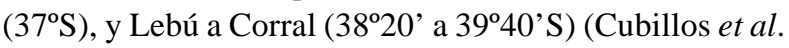
2005).

En la zona centro sur de Chile, entre los factores biológicos propuestos que afectarían la distribución de los primeros estadios tempranos de desarrollo de diversas especies de peces se incluyen aspectos biológicos relacionados con la reproducción de los adultos, tales como el tamaño de la hembra desovante (Landaeta 2007) y el patrón espacial de los desoves (Parada 1999, Parada et al. 2003). En el primer caso, el tamaño de las hembras desovantes puede determinar la ubicación del desove (e.g., hembras más grandes desovan más alejadas de la costa) o afectar las características propias del huevo y larva (i.e., tamaño de huevo, características bioquímicas y boyantez; Boyra et al. (2003), Llanos-Rivera (2005), Castro et al. (2009)) y en el segundo caso, la variabilidad espacial y temporal de los desoves puede depender de la selección de áreas con abundante alimento y condiciones ambientales óptimas (Fuiman 2002, Rodríguez-Graña \& Castro 2003, Lloret et al. 2004).

En el norte de Chile, los desoves de Engraulis ringens han sido asociados a bajas temperaturas del agua de mar, características de un evento de surgencia (Escribano et al. 1996), lo que influye sobre el desarrollo, crecimiento y las tasas metabólicas. La temperatura, la salinidad, oxígeno y el factor maternal influyen en el tamaño larval de eclosión y el tiempo de incubación (Sepúlveda et al. 2000, LlanosRivera 2005). En la zona centro sur de Chile, en cambio, los factores que determinarían la distribución de huevos y larvas no son tan claros, probablemente debido a que su distribución depende de múltiples factores relacionados con procesos de transporte y retención. Factores geográficos como la compleja batimetría de la zona podrían afectar la distribución de huevos y larvas de peces pelágicos. La presencia de terrazas y cañones sobre la plataforma continental, por ejemplo, contribuyen a variaciones en los tiempos de retención de estos estadios debido al desarrollo de estructuras de mesoescala capaces de retener o exportar huevos y larvas desde la costa (Arcos 1987, Vargas et al. 1996, Parada et al. 2001, Hormazabal et al. 2004, Morales et al. 2007).

También se ha observado que la ocurrencia de plumas de surgencia o convergencia de aguas oceánicas más cálidas sobre la plataforma, incluso durante el invierno, puede determinar variaciones en el hábitat de desove y finalmente la mortalidad de larvas al ser transportadas perpendicularmente a la costa desde donde fueron desovadas hacia áreas con menor concentración de alimento o con mayor abundancia de predadores (Castro et al. 2000, Castro \& Hernández 2000, Castro 2001). La presencia de áreas semi-cerradas como bahías y golfos pueden aumentar los tiempos de retención de estadios tempranos de invertebrados y peces que se desarrollan en su interior (Parada et al. 2001, Landaeta \& Castro 2002, Valle-Levinson et al. 2003) permitiéndole a las larvas crecer en un ambiente de alta concentración de alimento. Promontorios, puntas o quiebres en la línea de costa (i.e., Punta Lavapié) en las que se desarrollan surgencias, también se ha documentado que pueden conducir a modificaciones de los flujos en la zona costera y consecuentemente modular el transporte o retención de huevos y larvas en sus proximidades (Parada et al. 2001). Otros factores que pueden afectar la distribución de estadios tempranos de desarrollo de invertebrados y peces serían la presencia de desembocaduras de ríos, que además de generar plumas y estructuras de mesoescala como frentes halinos capaces de transportar organismos planctónicos fuera de la zona costera, pueden tener una gran influencia sobre las propiedades físicas, químicas del ambiente y sobre procesos biológicos en los organismos presentes en la región costera (Drinkwater \& Frank 1994). Estas variaciones en las propiedades del ambiente asociadas a las descargas de los ríos podrían modificar el crecimiento y mortalidad de los estadios tempranos de vida de peces debido a la escasa capacidad de regular o restaurar la presión osmótica que estos poseen (Drinkwater \& Frank 1994, Bunn et al. 2000, Grimes 2001, Quiñones \& Montes 2001).

En el área de desove de anchoveta y sardina común del 
centro-sur de Chile desembocan al menos tres ríos de mayor caudal: Itata (36 $26^{\circ}$ 'S), Biobío (36⒋'S) y Valdivia (3950’S). El presente estudio describió la distribución de huevos y larvas (preflexión notocordal) durante los periodos reproductivos principal (agosto-septiembre) y secundario (marzo), en el sector norte del área de desove centro sur de Chile, más particularmente en la zona adyacente a la desembocadura río Itata. El enfoque fue dirigido a determinar variaciones en la escala interanual tanto en distribución como en abundancia, en la última década (1996-2005).

\section{MATERIAL Y MÉtodos}

La información de la abundancia y distribución espacial de los huevos y larvas en preflexión de anchoveta y sardina común en la costa centro-sur de Chile, provienen de una serie de 7 cruceros oceanográficos realizados entre los $35,24^{\circ} \mathrm{S}$ y los $36,36^{\circ} \mathrm{S}$ (Tabla 1, Fig. 1). El área de interés incluye un sector al norte del río Itata, el área frente a la desembocadura, y una zona de menor extensión en el sector sur a la desembocadura. El área norte analizada fue de mayor extensión que su contraparte sur debido a que en el sector norte (proximidad a Punta Nugurne) se han encontrado históricamente altas concentraciones de huevos (Cubillos et al. 2005, 2006) y porque en el sector sur la distribución de huevos y larvas podrían confundirse con el efecto de otros ríos de mayor envergadura (i.e., río Biobío).

Los muestreos se realizaron perpendiculares a la costa, iniciándose a $0,5 \mathrm{mn}$ del litoral costero hasta a las $50 \mathrm{mn}$. En cada estación y transecta por medio, se llevaron a cabo perfiles hidrográficos con un CTD Seabird ${ }^{\circledR} 19$. Se utilizaron las mediciones de salinidad y temperatura de los diferentes años (2001, 2002, 2004, 2005), con el fin de poder encontrar una posible asociación de estas variables

Tabla 1. Periodo de muestreo, posición geográfica y número de estaciones de cada área de estudio / Sampling period, geographical location and number of stations in each study area

\begin{tabular}{|c|c|c|c|c|}
\hline \multicolumn{2}{|r|}{ Períodos } & \multicolumn{3}{|c|}{$\mathrm{N}^{\circ}$} \\
\hline Año & Mes & Coordenadas & Estaciones & Tipo red \\
\hline 1996 & Septiembre-Octubre & $35^{\circ} 40^{\prime} \mathrm{S}-36^{\circ} 30^{\prime} \mathrm{S}$ & 28 & Tucker trawl \\
\hline 2001 & Noviembre & $35^{\circ} 24^{\prime} \mathrm{S}-36^{\circ} 24^{\prime} \mathrm{S}$ & 36 & Tucker trawl \\
\hline 2002 & Marzo & $35^{\circ} 24^{\prime} \mathrm{S}-36^{\circ} 36^{\prime} \mathrm{S}$ & 42 & Tucker trawl \\
\hline 2002 & Agosto-Septiembre & $35^{\circ} 21^{\prime} \mathrm{S}-36^{\circ} 30^{\prime} \mathrm{S}$ & 187 & Calvet \\
\hline 2003 & Agosto-Octubre & $35^{\circ} 30^{\prime} \mathrm{S}-36^{\circ} 30^{\prime} \mathrm{S}$ & 79 & Calvet \\
\hline 2004 & Agosto-Septiembre & $35^{\circ} 34^{\prime} \mathrm{S}-36^{\circ} 30^{\prime} \mathrm{S}$ & 61 & Calvet \\
\hline 2005 & Septiembre & $35^{\circ} 34^{\prime} \mathrm{S}-36^{\circ} 30^{\prime} \mathrm{S}$ & 61 & Calvet \\
\hline Total & & & 494 & \\
\hline
\end{tabular}

con la distribución espacial de huevos y larvas de anchoveta y sardina común. Para estos análisis se utilizó la salinidad a $2 \mathrm{~m}$, debido a que a esa profundidad se espera observar la influencia de la pluma del río con mayor claridad. La utilización de valores de superficie no son normalmente recomendables, porque son altamente variables, producto de procesos de microescala o climáticos de corta duración.

Las muestras de ictioplancton en cada crucero fueron obtenidas mediante arrastres oblicuos con redes Tucker trawl ( $1 \mathrm{~m}^{2}$ área boca, $300 \mu \mathrm{m}$ trama, equipada con flujómetro digital General Oceanics) a profundidades cercanas a $50 \mathrm{~m}$, o mediante arrastres verticales con red Calvet $\left(0,05 \mathrm{~m}^{2}\right.$ área boca, $150 \mu \mathrm{m}$ trama $)$ desde profundidades cercanas a $70 \mathrm{~m}$ (Tabla 1 ) hasta superficie. El tamaño del ictioplancton colectado (diámetro de huevos de sardina $=0,98-1,02 \mathrm{~mm}$; diámetro menor de huevos de anchoveta $=0,5-0,7 \mathrm{~mm}$; diámetro de larvas de ambas especies 0,4-0,5 mm [en vista frontal, Moser \& Ahlstron 1985, Herrera et al. 1987]) fue mayor a las tramas utilizadas en las diferentes redes (Calvet $150 \mu \mathrm{m}$; Tucker trawl 300 $\mu \mathrm{m})$ por lo que procesos de extrusión son improbables. El volumen de agua de mar filtrada se obtuvo con la información del flujómetro digital para el caso de la red

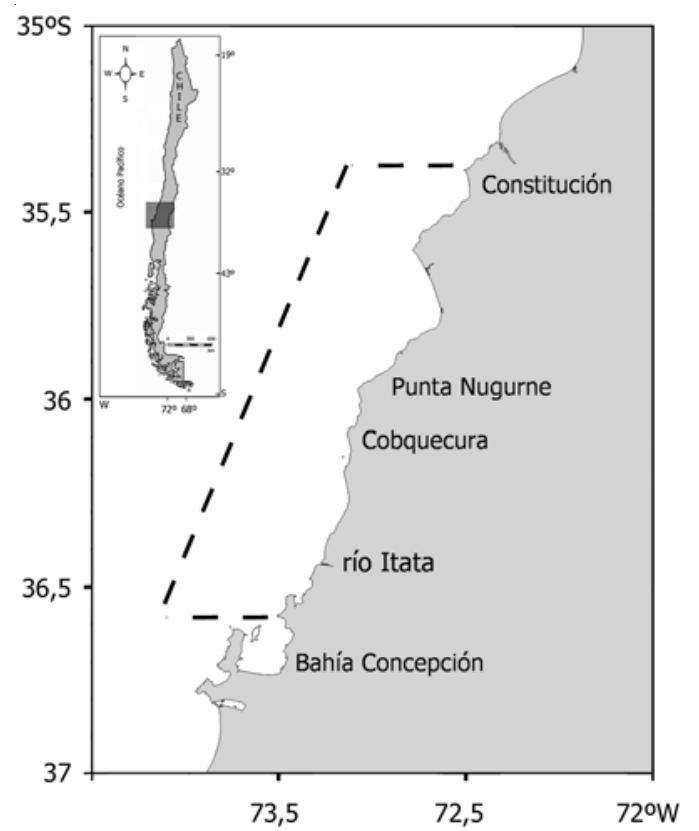

Figura 1. Área donde se desarrollaron los cruceros oceanográficos durante 1996-2005 en Chile central (línea segmentada) / Area surveyed during the oceanographic cruises from 1996 through 2005 in central Chile (dashed line) 
Tucker y mediante una estimación utilizando el área de la boca y profundidad del lance, para la red Calvet.

Las muestras de plancton fueron preservadas en una solución de formalina con agua de mar al 5\%, neutralizada con tetraborato de sodio (bórax). En laboratorio, se identificó y separó huevos y larvas de anchoveta y sardina común, utilizando los criterios de Moser \& Ahlstron (1985) y Herrera et al. (1987), clasificándose las larvas según su grado de desarrollo notocordal como en estado de pre y postflexión. La estandarización de la abundancia fue expresada en el número de individuos en $100 \mathrm{~m}^{-3}$.

El análisis de los datos generados incluyen i) diagramación espacial de abundancias de huevos y larvas de ambas especies en el área de estudio, ii) comparación inter-anual de abundancias de huevos y larvas por especie (ANDEVA, previo test de homogeneidad de varianzas), y iii) el uso de modelos aditivos generalizados (GAMs), como la herramienta principal para estudiar las relaciones entre la abundancia y las variables predictoras (latitud, periodo del crucero, salinidad, temperatura) ya que las relaciones esperadas son claramente no lineales. Los GAMs se ajustan a una amplia gama de formas funcionales y no requieren ningún supuesto respecto de la forma de las funciones (Agenbag et al. 2003). Un modelo aditivo permite al modelo lineal remplazar las funciones lineales de los predictores por funciones suavizadas. La forma general del modelo lineal es:

$$
Y=\alpha+\sum_{i=1}^{n} f_{i}\left(X_{i}\right)+\varepsilon
$$

donde $Y$ es la respuesta, $X_{i}$ es el predictor, $\alpha$ y $\beta$ son los parámetros y $\varepsilon$ es el error. La $f_{i}$ es generalmente desconocida y se estima en base a la dispersión de los datos (Agenbag et al. 2003), usando los programas Statistica 6.0 y $\mathrm{R}$ versión 2.6.1.

\section{Resultados}

\section{Presencia de huevos y larvas}

Los límites de variación en el porcentaje de estaciones positivas con huevos de anchoveta presentes en el área de estudio fueron muy diferentes (3-43\%), correspondiendo los menores valores hacia el final del periodo de desove (noviembre 2001). Durante los meses de máximo desove (julio-septiembre), el número de estaciones positivas con huevos fue mayor y el límite fue más estrecho (19-43\%). Los mayores porcentajes de estaciones positivas con presencia de huevos y de larvas de anchoveta ocurrieron el 2003 (huevos: 43\%) y el 2004 (larvas: 75\%), respectivamente.

En sardina común, el porcentaje de estaciones positivas fue inferior que en anchoveta, registrándose valores máximos del orden del 18\% para huevos y 29\% para larvas en 1996. Los mayores valores de estaciones positivas en ambas especies ocurrieron en los meses de agosto a septiembre (Tabla 2).

\section{VARIACIÓN EN ABUNDANCIA DE HUEVOS Y LARVAS}

Durante todos los años de estudio, los huevos de anchoveta fueron más abundantes que los de sardina común con un total de 838.223 huevos $100 \mathrm{~m}^{-3}$ y de 6.877 huevos $100 \mathrm{~m}^{-3}$

Tabla 2. Número de estaciones totales por periodo de estudio, estaciones con huevos y larvas preflexión de Engraulis ringens y Strangomera bentincki (estaciones positivas), y el porcentaje que representan las estaciones con huevos y larvas preflexión de cada especie del total de estaciones analizadas por periodo. (ND: no hay datos) / Total number of stations per sampling period, stations with eggs and preflexion larvae of Engraulis ringens and Strangomera bentincki (positive stations), and eggs and preflexion larval stations percentage in each species over all stations analyzed per period. (ND: no data)

\begin{tabular}{|c|c|c|c|c|c|c|c|c|c|c|}
\hline \multirow[b]{3}{*}{ Año } & \multirow[b]{3}{*}{ Mes } & \multirow[b]{3}{*}{$\begin{array}{c}\text { Estaciones } \\
\text { totales }\end{array}$} & \multicolumn{4}{|c|}{ ANCHOVETA } & \multicolumn{4}{|c|}{ SARDINA COMÚN } \\
\hline & & & \multicolumn{2}{|c|}{ Huevos } & \multicolumn{2}{|c|}{ Larva Preflexión } & \multicolumn{2}{|c|}{ Huevos } & \multicolumn{2}{|c|}{ Larva Preflexiór } \\
\hline & & & $\begin{array}{c}\text { Estaciones } \\
\text { positivas }\end{array}$ & $\%$ & $\begin{array}{c}\text { Estaciones } \\
\text { positivas }\end{array}$ & $\%$ & $\begin{array}{c}\text { Estaciones } \\
\text { positivas }\end{array}$ & $\%$ & $\begin{array}{c}\text { Estaciones } \\
\text { positivas }\end{array}$ & $\%$ \\
\hline 1996 & Septiembre-Octubre & 28 & 7 & 25 & 12 & 43 & 5 & 18 & 8 & 29 \\
\hline 2001 & Noviembre & 36 & 1 & 3 & 12 & 33 & 2 & 6 & 3 & 8 \\
\hline 2002 & Marzo & 42 & 15 & 36 & 30 & 71 & 5 & 12 & 9 & 21 \\
\hline 2002 & Agosto-Septiembre & 187 & 36 & 19 & 24 & 13 & 4 & 2 & 9 & 5 \\
\hline 2003 & Agosto-Octubre & 79 & 34 & 43 & ND & ND & 8 & 10 & ND & ND \\
\hline 2004 & Agosto-Septiembre & 61 & 18 & 30 & 46 & 75 & 5 & 8 & 5 & 8 \\
\hline 2005 & Septiembre & 61 & 13 & 21 & 32 & 52 & 2 & 3 & 8 & 13 \\
\hline
\end{tabular}


Tabla 3. Abundancia total de huevos $\left(100 \mathrm{~m}^{-3}\right)$ de anchoveta y sardina común en cada periodo de muestreo, promedio de huevos y desviación estándar (DE) sobre el total de estaciones y sólo sobre las estaciones positivas. Mínima y máxima abundancia de huevos en las estaciones por cada periodo de estudio / Total abundance $\left(100 \mathrm{~m}^{-3}\right)$ of anchoveta and common sardine eggs during each sampling period, mean egg and standard deviation (DE) over the total number of stations, and mean and standard deviations considering only the positive stations. Minimum and maximum of eggs abundance at the stations for each study period

\begin{tabular}{|c|c|c|c|c|c|c|c|c|}
\hline Año & Mes & Suma & $\begin{array}{l}\text { Promedio Total } \\
\text { estaciones }\end{array}$ & $\begin{array}{l}\text { DE Total } \\
\text { estaciones }\end{array}$ & $\begin{array}{l}\text { Promedio } \\
\text { Estaciones } \\
\text { Positivas }\end{array}$ & $\begin{array}{l}\text { DE Estaciones } \\
\text { Positivas }\end{array}$ & $\begin{array}{c}\text { Máxima } \\
\text { abundancia }\end{array}$ & $\begin{array}{c}\text { Minima } \\
\text { abundancia }\end{array}$ \\
\hline \multicolumn{9}{|c|}{ ANCHOVETA } \\
\hline 1996 & Septiembre-Octubre & 6426 & 229,5 & 766,8 & 918,0 & 1381,5 & 3810 & 2 \\
\hline 2001 & Noviembre & 363 & 10,1 & 60,4 & 362,5 & 0,0 & 363 & - \\
\hline 2002 & Marzo & 555741 & 13231,9 & 51895,0 & 37049,4 & 83315,4 & 299741 & 2 \\
\hline 2002 & Agosto-Septiembre & 14384 & 77,0 & 274,3 & 400,0 & 517,1 & 2800 & 25 \\
\hline 2003 & Agosto-Octubre & 229708 & 2908,7 & 9030,0 & 6756,1 & 12882,0 & 45102 & 29 \\
\hline 2004 & Agosto-Septiembre & 17454 & 286,1 & 1243,4 & 969,7 & 2180,5 & 9486 & 25 \\
\hline 2005 & Septiembre & 14147 & 231,9 & 1024,7 & 1088,2 & 2059,3 & 7509 & 27 \\
\hline \multicolumn{9}{|c|}{ SARDINA COMUN } \\
\hline 1996 & Septiembre-Octubre & 461 & 16,5 & 51,1 & 92,2 & 94,3 & 233 & 3 \\
\hline 2001 & Noviembre & 393 & 10,9 & 52,1 & 196,3 & 149,4 & 302 & 91 \\
\hline 2002 & Marzo & 503 & 12,0 & 42,8 & 100,5 & 87,4 & 234 & 12 \\
\hline 2002 & Agosto-Septiembre & 1274 & 7,0 & 51,4 & 318,0 & 177,5 & 541 & 133 \\
\hline 2003 & Agosto-Octubre & 3145 & 39,8 & 225,5 & 393,1 & 529,5 & 1545 & 50 \\
\hline 2004 & Agosto-Septiembre & 830 & 13,6 & 64,9 & 166,0 & 177,5 & 467 & 31 \\
\hline 2005 & Septiembre & 271 & 4,4 & 25,4 & 135,3 & 56,7 & 175 & 95 \\
\hline
\end{tabular}

Tabla 4. Abundancia total de larvas $\left(100 \mathrm{~m}^{-3}\right)$ de anchoveta y sardina común en cada periodo de muestreo, promedio de larvas y desviación estándar (DE) sobre el total de estaciones, y sólo sobre las estaciones positivas. Mínima y máxima abundancia de larvas en las estaciones por cada periodo de estudio / Total abundance $\left(100 \mathrm{~m}^{-3}\right)$ of anchoveta and common sardine larvae during each sampling period, mean larval and standard deviation (DE) over the total number of stations, only in the positive stations. Minimum and maximum larvae abundance at the stations for each study period

\begin{tabular}{|c|c|c|c|c|c|c|c|c|}
\hline Año & Mes & Suma & $\begin{array}{l}\text { Promedio Total } \\
\text { estaciones }\end{array}$ & $\begin{array}{l}\text { DE Total } \\
\text { estaciones }\end{array}$ & $\begin{array}{c}\text { Promedio Estaciones } \\
\text { Positivas }\end{array}$ & $\begin{array}{l}\text { DE Estaciones } \\
\text { Positivas }\end{array}$ & $\begin{array}{c}\text { Máxima } \\
\text { abundancia }\end{array}$ & $\begin{array}{c}\text { Minima } \\
\text { abundancia }\end{array}$ \\
\hline
\end{tabular}

\section{ANCHOVETA}

$\begin{array}{ll}1996 & \text { Septiembre-Octubre } \\ 2001 & \text { Noviembre } \\ 2002 & \text { Marzo } \\ 2002 & \text { Agosto-Septiembre } \\ 2003 & \text { Agosto-Octubre } \\ 2004 & \text { Agosto-Septiembre } \\ 2005 & \text { Septiembre }\end{array}$

$\begin{array}{rrcr}11 & 0,4 & 0,6 & 1,1 \\ 784 & 21,8 & 63,3 & 65,4 \\ 88750 & 2113,1 & 4082,2 & 2958,3 \\ 1532 & 7,7 & 28,0 & 63,8 \\ \mathrm{ND} & \mathrm{ND} & \mathrm{ND} & \mathrm{ND} \\ 12859 & 210,8 & 332,1 & 279,5 \\ 7911 & 129,7 & 252,3 & 247,2\end{array}$

\section{SARDINA COMUN}

$\begin{array}{ll}1996 & \text { Septiembre-Octubre } \\ 2001 & \text { Noviembre } \\ 2002 & \text { Marzo } \\ 2002 & \text { Agosto-Septiembre } \\ 2003 & \text { Agosto-Octubre } \\ 2004 & \text { Agosto-Septiembre } \\ 2005 & \text { Septiembre }\end{array}$

24
303
2328
353
$\mathrm{ND}$
160
987

$\begin{array}{ccc}0,8 & 2,7 & 3,1 \\ 8,4 & 40,9 & 101,0 \\ 55,4 & 199,1 & 259,0 \\ 1,8 & 8,6 & 39,3 \\ \mathrm{ND} & \mathrm{ND} & \mathrm{ND} \\ 2,6 & 9,6 & 32,1 \\ 16,2 & 75,1 & 123,4\end{array}$

$\begin{array}{rrr}0,5 & 2 & 1 \\ 98,3 & 300 & 1 \\ 4579,6 & 21877 & 1 \\ 51,5 & 267 & 27 \\ \mathrm{ND} & \mathrm{ND} & \mathrm{ND} \\ 357,0 & 1467 & 25 \\ 305,2 & 1200 & 27\end{array}$

ND: No hay datos 
respectivamente (Tabla 3). Las máximas abundancias de huevos fueron 37.049 huevos $100 \mathrm{~m}^{-3} \mathrm{y} 6.756$ huevos 100 $\mathrm{m}^{-3} \mathrm{y}$ se registraron en marzo (2002) y en agosto-septiembre (2003) respectivamente. En sardina común, la mayor abundancia se observó en los meses de agosto-septiembre (318 huevos $100 \mathrm{~m}^{-3}$ en 2002 y 393 huevos $100 \mathrm{~m}^{-3}$ en 2003) (Tabla 3).

Las larvas de anchoveta también fueron más abundantes que las de sardina común. La abundancia total para larvas de anchoveta fue de 111.847 larvas $100 \mathrm{~m}^{-3} \mathrm{y}$ la de sardina común fue de 4.155 larvas $100 \mathrm{~m}^{-3}$ (Tabla 4). Las mayores abundancias de ambas especies (>100 larvas $100 \mathrm{~m}^{-3}$ ) se encontraron en los meses de marzo (2002) y agostoseptiembre (2004-2005) (Tabla 4).

La abundancia de huevos y larvas de anchoveta mostró grandes fluctuaciones durante el periodo de estudio, con diferencias significativas entre cruceros $\left(\mathrm{F}_{(6,487)}=4,59, P\right.$ $<0,05$ huevos, $\mathrm{F}_{(5,409)}=18,99, P<0,001$ larvas). Sin embargo, descontando los valores de abundancia observados en agosto-septiembre del 2003 en que se cuantificó altas abundancias, el resto de los años presentó valores de abundancia similares entre si durante el periodo de mayor actividad reproductiva (Fig. 2a). En sardina común, también se presentaron cambios significativos en la abundancia de los estadios tempranos entre los distintos años $\left(\mathrm{F}_{(6,487)}=2,19, P<0,05\right.$ huevos, $\mathrm{F}_{(5,409)}=4,29, P<$ 0,001 larvas). Sin embargo, y a diferencia de la anchoveta, al principio de los años 2002 y 2003 se observaron años de mayor abundancia, para posteriormente disminuir en el tiempo (2004-2005) (Fig. 2b). La abundancia de larvas preflexión de esta especie, alternativamente, tendió a aumentar desde el 2004 al 2005 (Fig. 2b).

\section{DisTRIBUCIÓN DE HUEVOS Y LARVAS}

Las mayores abundancias de huevos de anchoveta se observaron en el sector norte del área de estudio $\left(35,3^{\circ} \mathrm{S}\right.$ a $35,7^{\circ} \mathrm{S}$ ) en todos los años, excepto el año 2005 en que se presentaron en el sector sur $\left(36,1^{\circ} \mathrm{S}\right.$ a $\left.36,3^{\circ} \mathrm{S}\right)$. La mayor cobertura de huevos se presentó en el año 2003 alcanzando una distancia máxima de $50 \mathrm{mn}$ de la costa. En los años restantes, la distribución de huevos fue más costera ( 10 a $20 \mathrm{mn}$ de la costa). Las mayores abundancias de larvas de un año a otro variaron espacialmente, sin un patrón latitudinal claro (Fig. 3). La distribución costa-océano de larvas en los diferentes años tendió a cubrir una superficie mayor del área de estudio que la de huevos, llegando incluso a las $50 \mathrm{mn}$ de la costa. En los años 2002, 2004 y 2005 se observaron altas abundancias incluso hasta en las estaciones más alejadas de la costa.

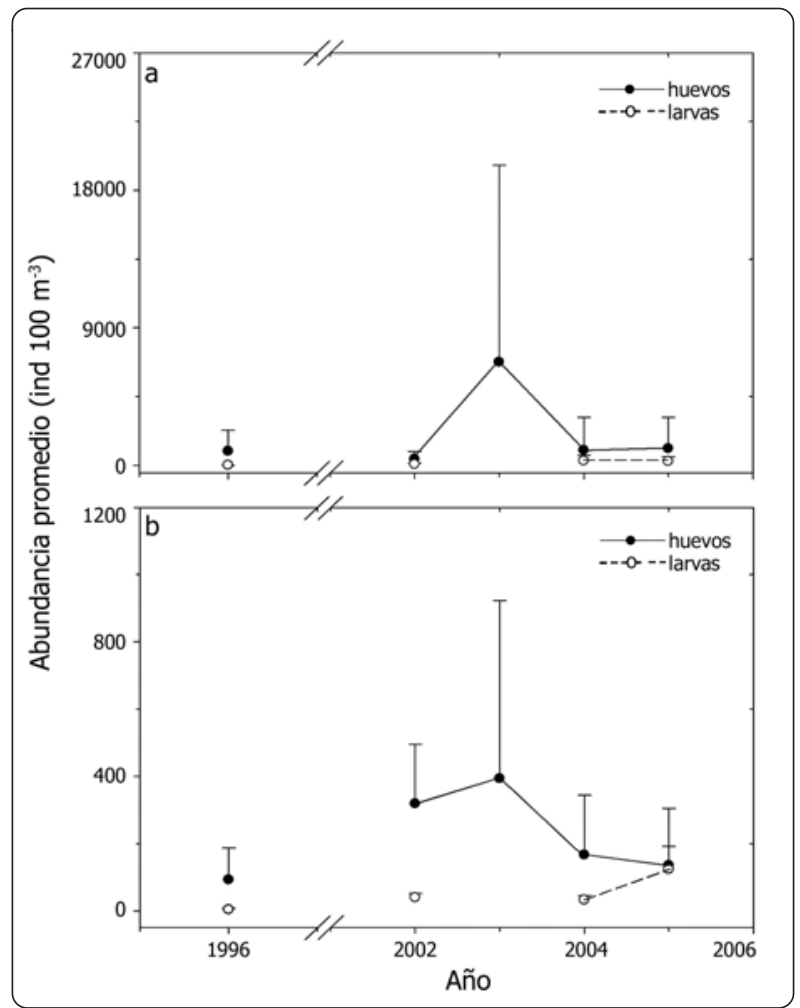

Figura 2. Abundancia promedio (ind $100 \mathrm{~m}^{-3}$ considerando solo las estaciones positivas (ver tablas 3 y 4 ) de huevos y larvas de anchoveta (a) y sardina común (b) correspondiente al periodo 1996 a 2005. Los años corresponden a muestreos sólo en los meses del máximo reproductivo (julio-septiembre) / Mean abundance (ind $100 \mathrm{~m}^{-3}$, considering only positive stations, see tables 3 and 4) of anchoveta (a) and common sardine (b) eggs and larvae from 1996 to 2005. Data are the samplings carried out only during the peak reproductive month (july-september)

Concordando con los análisis espaciales que señalan una variación interanual en las distribuciones de huevos y larvas, el ajuste de los modelos Aditivos Generalizados (GAMs) mostraron que las variables temporales (periodo del crucero) y espaciales (latitud) estarían describiendo adecuadamente la abundancia de huevos y larvas tanto para anchoveta como sardina común (Tabla 5).

En el sector de la desembocadura del río Itata hubo presencia de huevos en todos los años, excepto el año 2001 (Fig. 4). En los años 1996 y 2004, agrupaciones de huevos con relativamente altas abundancias fueron encontradas en la proximidad a la desembocadura ( $>250$ huevos 100 $\left.\mathrm{m}^{-3}\right)$. En esta zona, se observaron larvas prácticamente en todos los años (excepto el 2001) pero con abundancias variables (50 a 500 larvas $100 \mathrm{~m}^{-3}$ ) (Fig. 5).

En sardina común, latitudinalmente las mayores abundancias de huevos y larvas se presentaron en el sector 


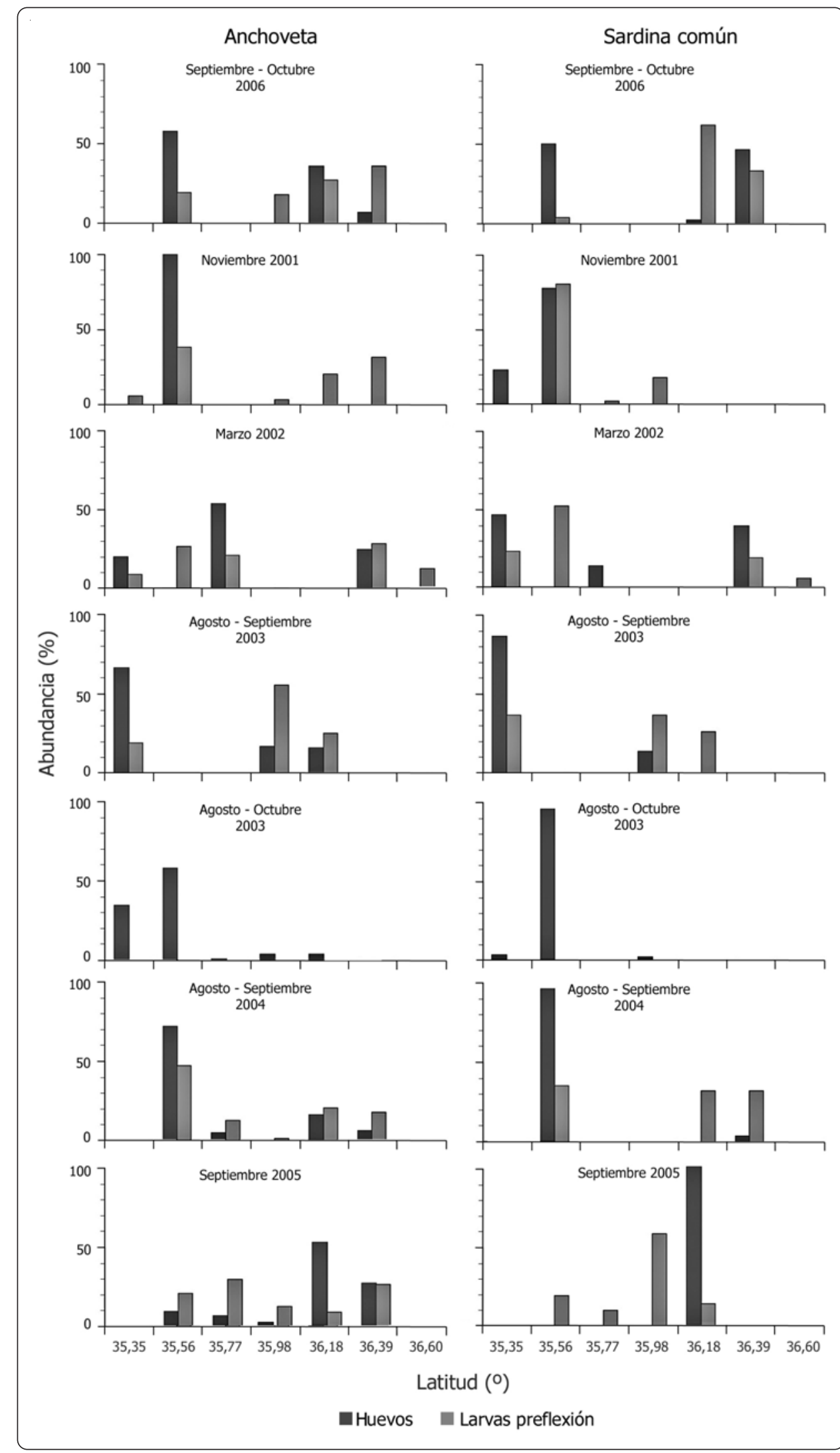

Figura 3. Variación latitudinal y temporal de la abundancia promedio de huevos y larvas en preflexión para anchoveta y sardina común (\%). Porcentaje corresponde a la suma de las abundancias contenidas en las estaciones a una latitud determinada / Latitudinal and temporal variations in mean of anchoveta (a) and common sardine (b) eggs and preflexion larvae (\%). Percentage is obtained from the addition of abundances in all stations at a same latitude 


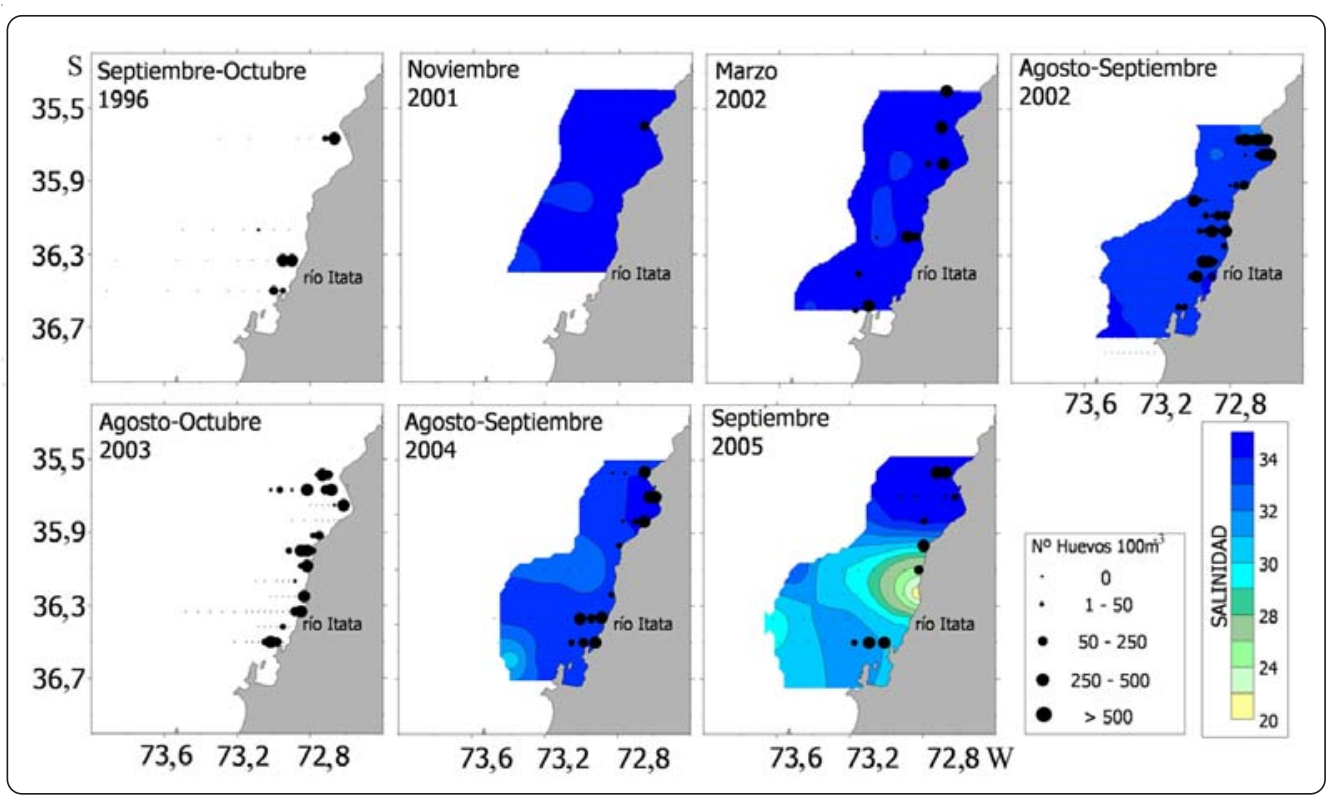

Figura 4. Distribución de huevos de Engraulis ringens sobre secciones horizontales de salinidad a $\sim 2 \mathrm{~m}$ de profundidad entre 1996 a 2005 / Engraulis ringens egg distributions overimpossed on salinity horizontal sections at $\sim 2$ m depth between 1996 and 2005

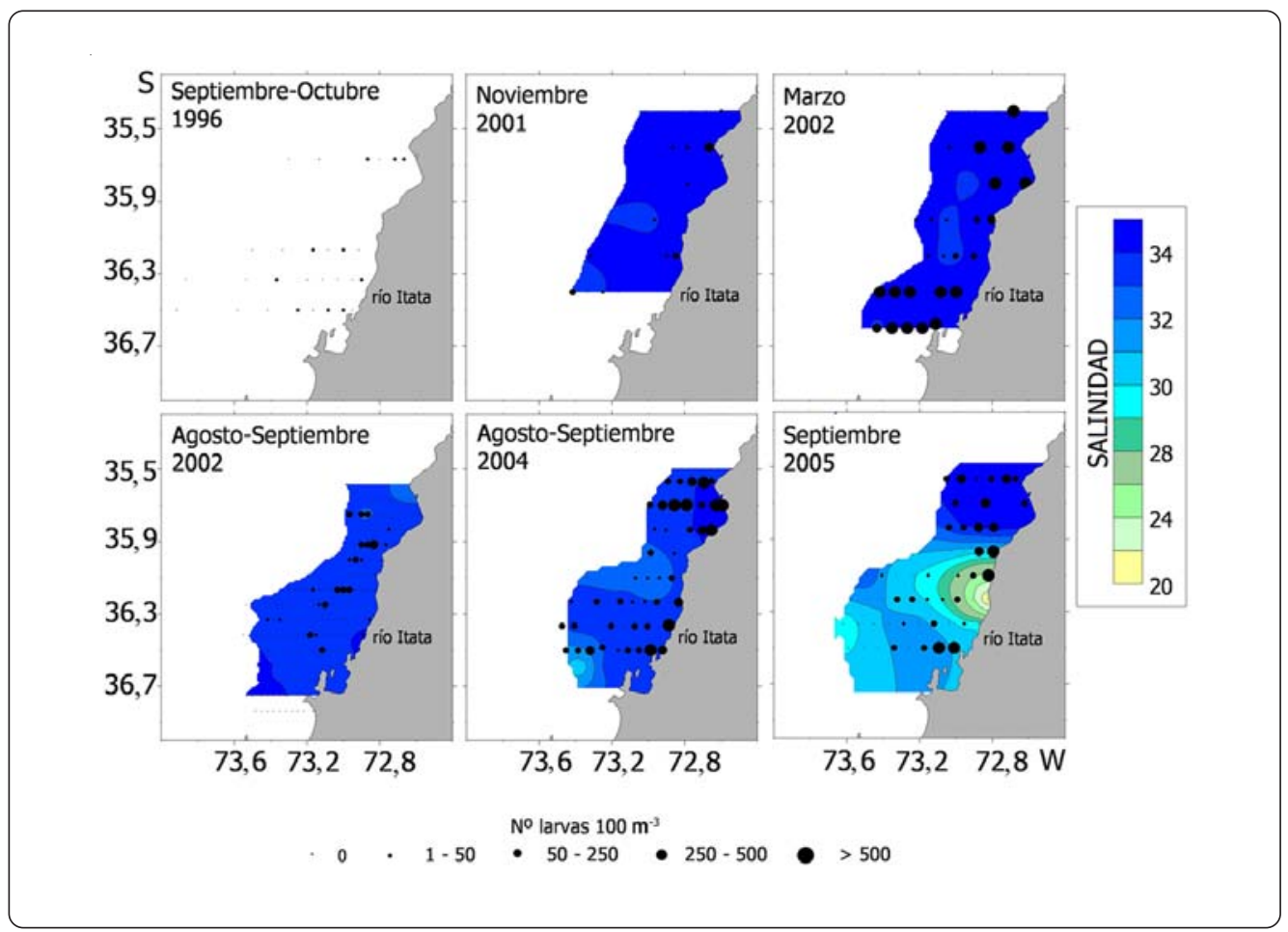

Figura 5. Distribución de larvas en preflexión de Engraulis ringens sobre secciones horizontales de salinidad a $\sim 2$ m de profundidad entre 1996 a 2005 / Engraulis ringens preflexion larvae distributions overimpossed on salinity horizontal sections at $\sim 2 \mathrm{~m}$ depth between 1996 and 2005 
Tabla 5. Resumen de los resultados de los GAMs utilizados en la distribución espacial y temporal de huevos y larvas en preflexión de anchoveta y sardina común / Summary of GAMs results used in the spatial and temporal distribution of eggs and preflexion larvae of anchoveta and common sardine

\begin{tabular}{lcrrc}
\hline \multicolumn{1}{c}{ Estados por especies } & Periodo de crucero & Latitud & Temperatura & Salinidad \\
\hline Huevos anchoveta & $3,159^{*}$ & $5,636^{*}$ & $5,538^{*}$ & $2,756 *$ \\
Larva preflexión anchoveta & $1,913^{*}$ & $13,578^{*}$ & $3,570 *$ & $1,578 *$ \\
Huevos sardina común & $3,087^{*}$ & $2,620 *$ & $0,759 *$ & $1,233 *$ \\
Larva preflexión sardina común & $0,982 *$ & $4,186 *$ & $0,186 *$ & $0,086 *$ \\
\hline
\end{tabular}

Valores de los parámetros (periodo crucero (año), latitud, temperatura, salinidad). Los valores significativos $(P<0,05)$ se indican con *

norte $\left(35,3^{\circ} \mathrm{S}\right.$ a $\left.35,9^{\circ} \mathrm{S}\right)$, con excepción del 2005 que se ubicaron en el sector sur del área de estudio $\left(35,9^{\circ} \mathrm{S}\right.$ a $36,1^{\circ} \mathrm{S}$ ) (Fig. 3). Longitudinalmente, la distribución fue principalmente costera (10 mn aprox.). En el sector de la desembocadura del río Itata, los huevos ocurrieron solamente en los años 1996, 2002 (marzo) y 2005 pero en baja abundancia (Fig. 6). En larvas, la distribución fue ocasionalmente más amplia que en huevos (hasta $50 \mathrm{mn}$ de la costa aproximadamente), presentándose abundancias más bajas (1-50 larvas $100 \mathrm{~m}^{-3}$ ) en las zonas más alejadas de la costa. En el sector de la desembocadura del río Itata, la presencia de larvas se restringió al año 2002 (Fig. 7).

\section{Abundancia de huevos y larvas en relación a la SALINIDAD}

Los resultados de los valores de los parámetros obtenidos por los GAMs tanto para anchoveta como sardina común (Tabla 5) muestran que las relaciones no lineales entre la variable respuesta (abundancia) y predictor (salinidad) son significativas $(P<0,05)$, es decir que a ciertas salinidades (30-34 ups) presentaron las mayores abundancias.

Espacialmente, las agrupaciones de huevos de anchoveta se encontraron a salinidades entre los 32 a 34 ups en los años 2001, 2002 y 2004. En cambio para el año 2005, el rango de salinidad fue entre los 20 a 34, y con una mayor ocurrencia de huevos en salinidades desde 24 a 34 ups (Fig. 4). En larvas, las agrupaciones durante los años 2001, 2002 y 2004 se encontraron en salinidades entre los 30 a 34 ups, excepto para el año 2005 donde las mayores ocurrencias de larvas se situaron en salinidades entre los 24 a 34 ups (Fig. 5), al igual que los huevos.

Los huevos de sardina común se hallaron en salinidades entre los 33 y 34 ups en los años 2001, 2002 y 2004, sin embargo para el año 2005 ocurrieron también en salinidades bajas (<29 ups) (Fig. 6). Las larvas se encontraron en salinidades entre los 30 y 34 ups, excepto en el año 2005 donde el rango de salinidad fue más amplio (23 a 34 ups) (Fig. 7). Adicionalmente, en el año 2004 se presentó una masa de agua de baja salinidad (29-32 ups) fuera de la costa frente del sector de Bahía Concepción, donde se encontraron pequeñas agrupaciones de larvas de anchoveta y sardina común (Fig. 5 y 7)

$\mathrm{Al}$ analizar la transecta frente a la desembocadura del río Itata (costa-océano) durante todos los años (excepto el 2001 y 2002 en que no se encontró huevos ni larvas de sardina común en la transecta), se observó que las mayores abundancias de huevos de anchoveta y sardina común fueron más costeras ( $<10 \mathrm{mn}$ ) en comparación a las larvas (>10 mn) (Fig. 8). Dentro de las 10 primeras millas náuticas se observa un ascenso de las isohalinas de 34 y 34,2 ups, excepto en el año 2005, donde en superficie encontramos salinidades inferiores a 30 ups. La isohalina de 34,4 ups en los años 2001 y 2002 se ubicó en aguas más someras (menor a $40 \mathrm{~m}$ de profundidad), en cambio para los años 2004 y 2005 fue más profunda (45 a 100 m). La isohalina de 34 ups en los años 2001, 2002 y 2004 se presentó sobre los $25 \mathrm{~m}$ de profundidad, excepto en el 2005 donde se ubicó entre los 50 y 60 m (Fig. 8).

Para ver si existían variaciones en abundancia entre la desembocadura (distancia $=0 \mathrm{mn}$ ) (Fig. 9) y las zonas norte y sur adyacentes, se diagramó una transecta costera que se extendió $10 \mathrm{mn}$ hacia el sur y hasta $50 \mathrm{mn}$ hacia el norte. En el área entre la desembocadura y las $10 \mathrm{mn}$, se encontró una baja abundancia de huevos y larvas de anchoveta y sardina común, excepto en el año 2002 ( 8.500 huevos $\left.100 \mathrm{~m}^{-3}\right)$ y $2004\left(\sim 2.500\right.$ huevos $\left.100 \mathrm{~m}^{-3}\right)$. Entre las $11 \mathrm{a}$ $40 \mathrm{mn}$ se hallaron las mayores abundancias de ambos estadios y en ambas especies $\left(<350.000\right.$ ind $\left.100 \mathrm{~m}^{-3}\right)$. En anchoveta, la mayor abundancia de huevos ( $>300.000$ huevos $\left.100 \mathrm{~m}^{-3}\right)$ y larvas $\left(\sim 4.500\right.$ larvas $\left.100 \mathrm{~m}^{-3}\right)$ se encontró en el 2002 a una distancia de $35 \mathrm{mn}$ con respecto 


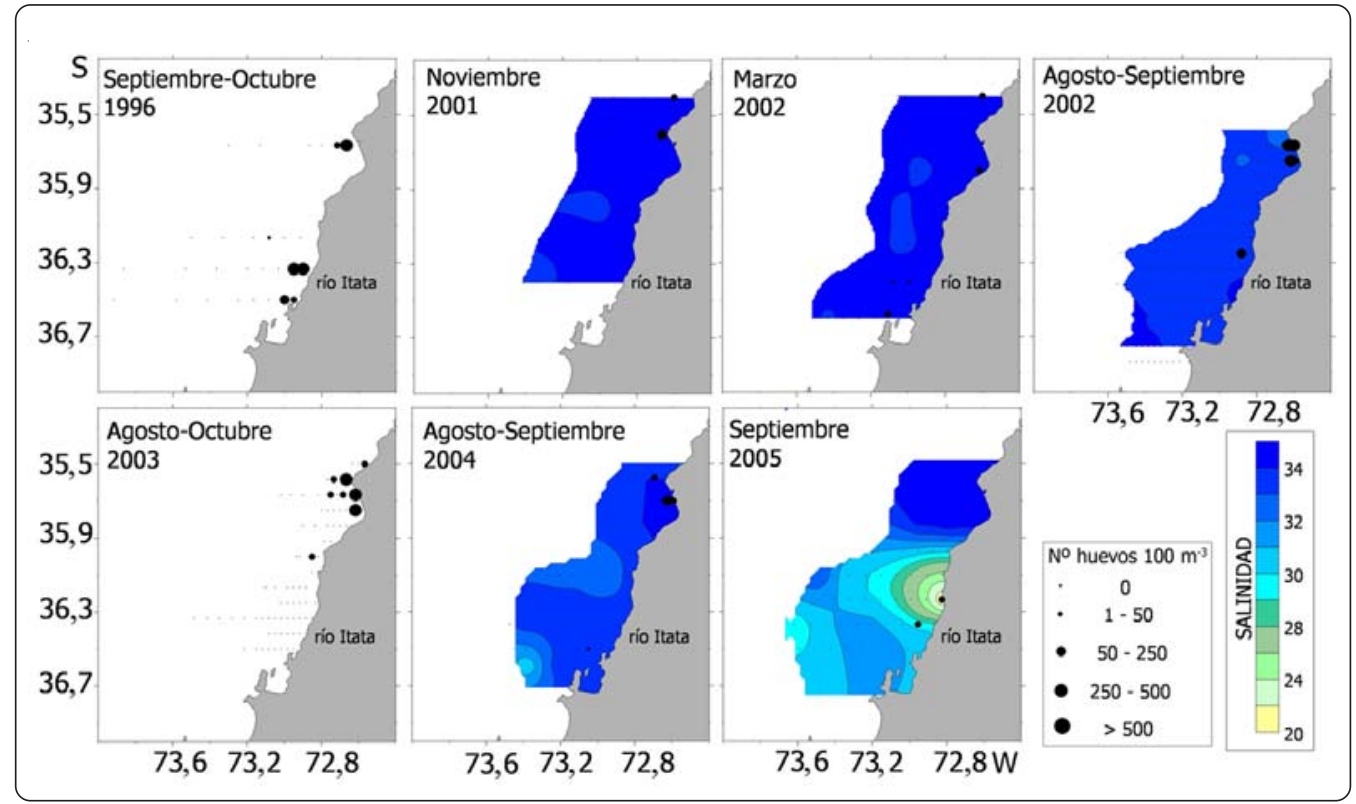

Figura 6. Distribución de huevos de $\mathrm{S}$. bentincki sobre secciones horizontales de salinidad a $\sim 2 \mathrm{~m}$ de profundidad entre 1996 a 2005 / S. bentincki egg distributions overimpossed on salinity horizontal sections at $\sim 2 \mathrm{~m}$ depth between 1996 and 2005

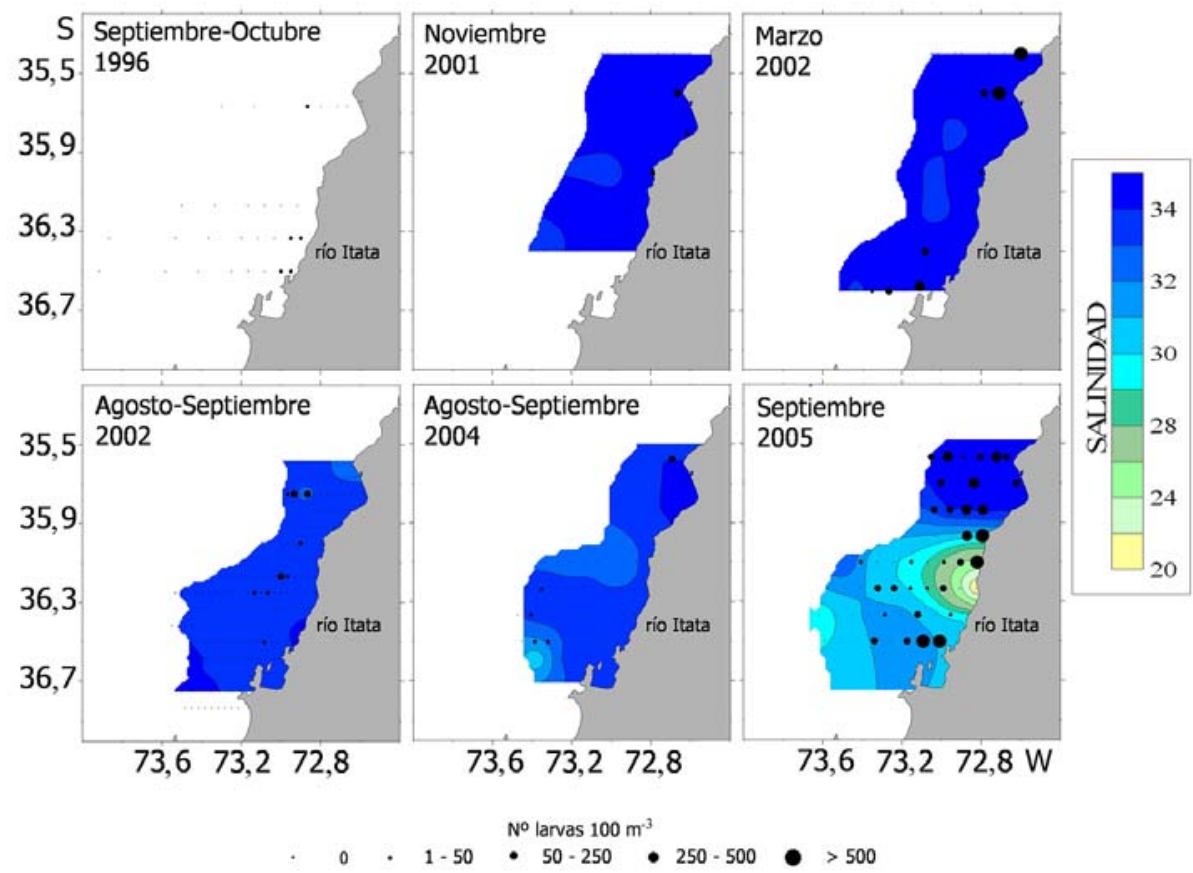

Figura 7. Distribución de larvas en preflexión de S. bentincki sobre secciones horizontales de salinidad a $\sim 2 \mathrm{~m}$ de profundidad entre 1996 a 2005 / S. bentincki preflexion larvae distributions overimpossed on salinity horizontal sections at $\sim 2 \mathrm{~m}$ depth between 1996 and 2005 
a la desembocadura. En sardina común se encontró una baja abundancia de huevos y larvas ( $<700$ ind $100 \mathrm{~m}^{-3}$ ), pero su distribución fue similar a la descrita para la anchoveta. Con respecto a la distancia máxima muestreada (50 mn) los valores de abundancia fueron bajos (< 750 ind $100 \mathrm{~m}^{-3}$ ) en ambas especies (Fig. 9).

En la sección vertical de salinidad se observa que a medida que nos alejamos de la desembocadura los valores tienden a incrementarse, es decir, las isohalinas que cortan la superficie dentro las $10 \mathrm{mn}$ presentaron valores que fluctuaron entre los 29 a 33,8 ups, mientras que las isohalinas que cortan la superficie entre las 30 a $40 \mathrm{mn}$ presentaron valores altos (34,2-34,4-34,5 ups). Durante el 2004 se observó un ascenso ( 20 a 10 m) de las isohalinas (34-34,2 ups) en dirección a la desembocadura. En el 2005, al norte de la desembocadura se presentó un frente halino muy marcado, cuyos valores de salinidad en superficie fluctuaron entre los 29 a 34 ups (Fig. 9).

\section{Abundancia de huevos y larvas en relación a la TEMPERATURA}

Los resultados de los valores de ajuste de los GAMs tanto para anchoveta como sardina común (Tabla 5) indicaron que la relación entre la variable respuesta (abundancia) y predictor (temperatura) son significativas $(P<0,05)$, es decir que a ciertas valores de temperaturas $\left(10-11^{\circ} \mathrm{C}\right)$ se presentaron las mayores abundancias de huevos y larvas de anchoveta; en sardina común estos valores fluctuaron entre 11,5 y $12^{\circ} \mathrm{C}$.

\section{Discusión}

Las poblaciones de pequeños peces pelágicos como sardina común y anchoveta que habitan en los sistemas de surgencia de la región centro-sur de Chile han presentado grandes variaciones en su biomasa (i.e., 4,3 a 1,8 millones de toneladas, Oliva et al. (2002)). Tales fluctuaciones normalmente se atribuyen a factores exógenos y endógenos que afectan la sobrevivencia y distribución de huevos y larvas, lo que posteriormente se refleja en el éxito o fracaso del reclutamiento (Grimes \& Finucane 1991, Dorsey et al. 1996, Fuiman 2002, Lloret et al. 2004). Dentro de la zona de desove centro sur de Chile, sin embargo, la distribución del desove no es homogénea sino que algunas zonas presentan mayores concentraciones de huevos y larvas. En el presente estudio documentamos variaciones tanto espaciales como temporales de abundancia de huevos y larvas de anchoveta y sardina común en el área próxima a la desembocadura del río Itata y la zona norte adyacente a ésta.

\section{DistribuCión de HUEVOS Y LARVAS}

Nuestros análisis de distribución de estadios de desarrollo temprano muestran que los huevos de anchoveta y sardina común fueron más abundantes en la franja costera, principalmente en el norte de la zona de estudio para el caso de anchoveta, lo cual coincide con los antecedentes reportados por Nuñez et al. (1995) para la costa de la VIII Región (36-37 $\mathrm{S})$, quienes mencionan que las mayores densidades de huevos y larvas de ambas especies se asocian a la terraza del Itata y al Golfo de Arauco ( $<10 \mathrm{mn}$ ), en muestreos de hasta las $25 \mathrm{mn}$ de la costa. La distribución de las larvas del presente estudio fue más amplia (hasta $50 \mathrm{mn}$ de la costa) en comparación a la de los huevos, presentando las mayores densidades tanto a nivel costero como oceánico (50 mn). La ubicación de las mayores densidades de huevos de anchoveta y sardina común observadas en este estudio concuerdan con la información de zonas de desove de anchoveta localizadas en el área centro sur de Chile, área en que se ha señalado que los huevos de anchoveta y sardina común ocurren

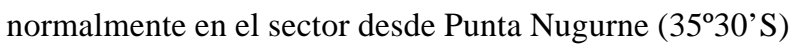
hasta la desembocadura del río Itata durante los meses de mayor actividad reproductiva (Cubillos et al. 2005, 2006).

Para meses de baja actividad reproductiva (i.e., octubrediciembre, fines de estación reproductiva principal) o para el periodo secundario de desove que se inicia a fines del verano (febrero-marzo) de cada año (Cubillos \& Arancibia 1993, Arcos et al. 1996), la información de distribución de huevos y larvas de anchoveta y sardina común es muy escasa para la zona adyacente a la desembocadura del río Itata. Nuestros resultados señalan que en noviembre 2001 se encontraron huevos de anchoveta sólo en una estación en el sector más al norte y que sus larvas (Fig. 3) ocurrieron en muy baja abundancia pero más dispersas en el área de estudio. En marzo 2002 (fines del máximo secundario de desove), los huevos de anchoveta ocurrieron en bajas abundancias y muy espaciados a lo largo de toda la franja costera, contrastando con las altas abundancias de larvas de esta especies que, además, se distribuyeron ampliamente en la zona de estudio con concentraciones mayores tanto en el sector norte como en el sector sur donde llegaron hasta las estaciones más alejadas de la costa. Durante noviembre del 2001 y marzo del 2002, los huevos y larvas de sardina común siguieron los mismos patrones de distribución que los de anchoveta, pero presentando valores de abundancia menores. Respecto a los cambios de abundancia de huevos, se desconoce el número de huevos producidos por cada especie en noviembre 2001 y marzo 2002, sin embargo, información de los años siguientes 


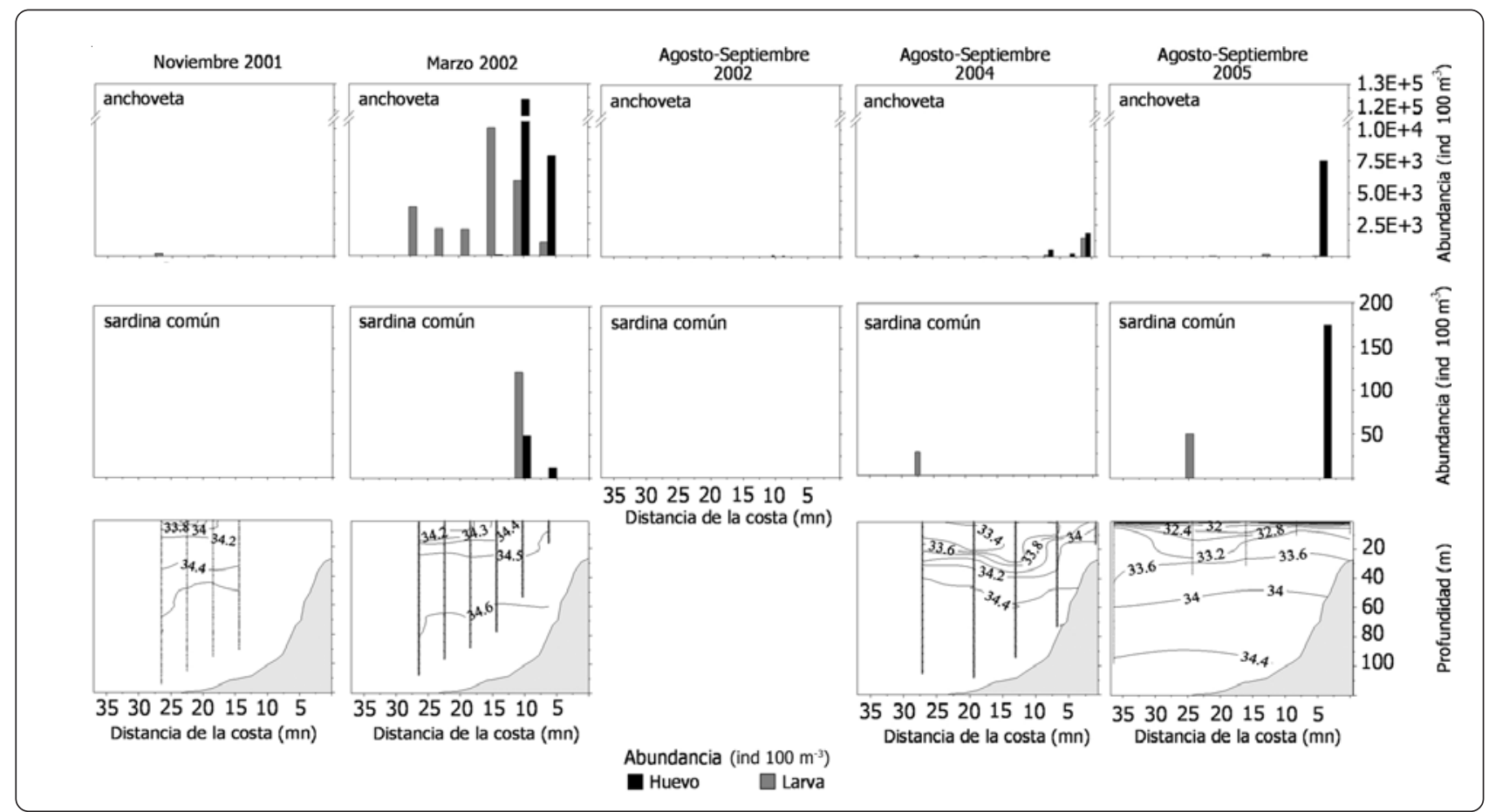

Figura 8. Distribución (costa-océano: este-oeste) de los estadios tempranos de vida de anchoveta y sardina común y secciones verticales de salinidad frente a la desembocadura del río Itata $(\mathbf{0} \mathbf{~} \mathbf{n})$ / Distribution (coast-ocean: east-west) of early life stages of anchoveta and common sardine and vertical salinity sections off Itata River mouth ( 0 nautical miles in the figure)

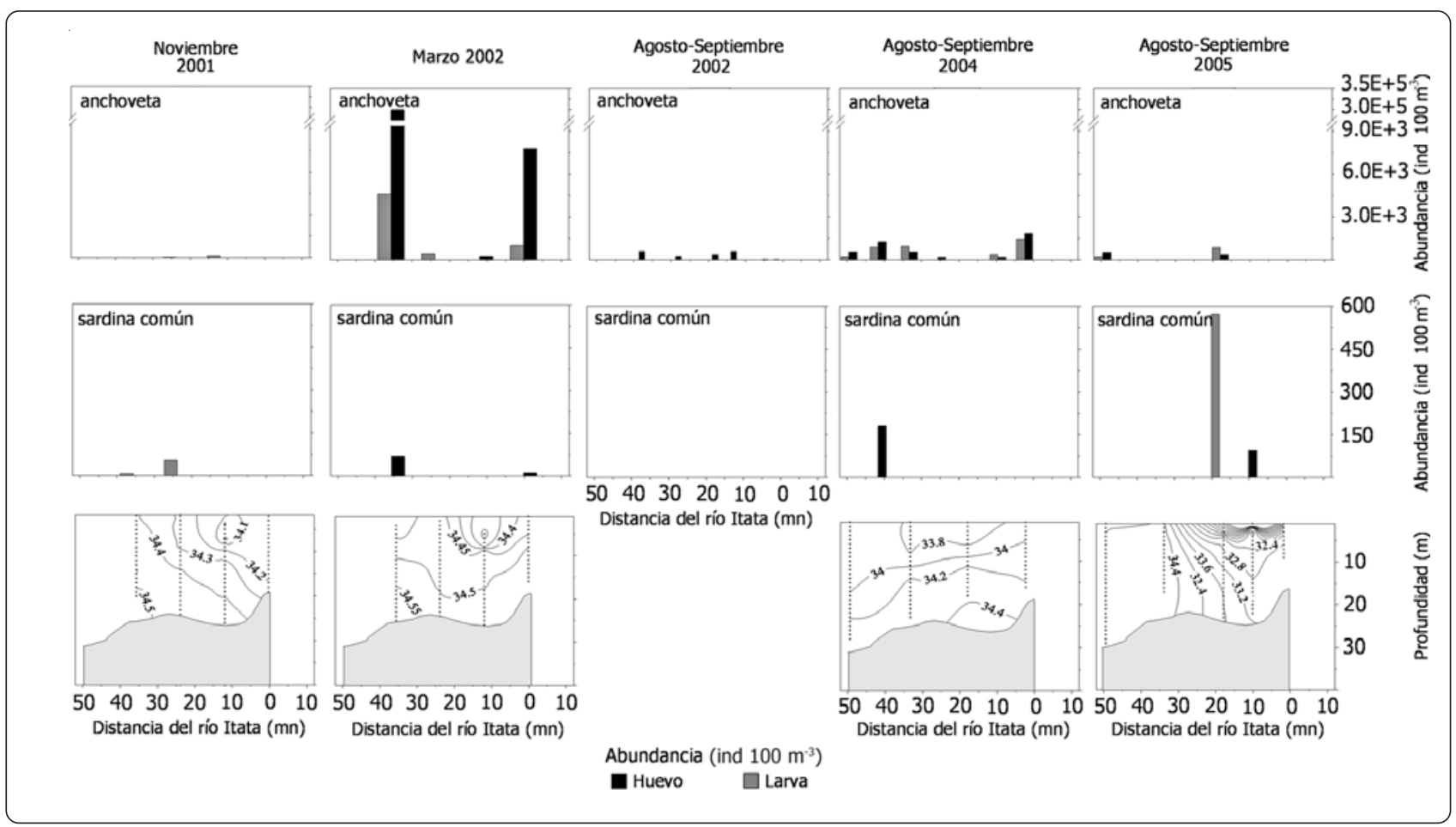

Figura 9. Distribución (transecta costera: norte-sur) de los estadios tempranos de vida de anchoveta y sardina común y secciones verticales de salinidad en el área adyacente a la desembocadura del río Itata (0 $\mathbf{~ m n}$ ) / Distribution (coastal transect: north south) of early life stages of anchoveta and common sardine and vertical sections of salinity in the adjacent area of Itata River mouth ( 0 nautical miles) 
(Bernal et al. 2004, Cubillos et al. 2005) señalan que la producción de huevos de sardina común sería menor que la de anchoveta en la zona de estudio, concordando entonces con la diferencia en abundancia de huevos y larvas entre especies de nuestro estudio.

\section{Abundancia de huevos y larvas en relación a la TEMPERATURA}

La temperatura presenta una relación positiva entre los $10^{\circ}$ a $11^{\circ} \mathrm{C}$ con la abundancia de huevos y larvas de anchoveta. Para la sardina común esta relación se da a temperaturas más altas y en un rango más estrecho de temperatura $\left(11,5^{\circ}\right.$ a $12^{\circ} \mathrm{C}$ ). Estas relaciones concuerdan con lo citado por Escribano et al. (1996) para el norte de Chile y por Cubillos et al. (2001) y Cubillos et al. (2005) en la zona centro sur, en cuanto a que las mayores abundancias de huevos de anchoveta ocurren en aguas de menores temperaturas dentro de las respectivas zonas durante el desove. Ambas especies incluyen en su estrategia reproductiva el desovar al término del invierno (agosto), cuando existen las condiciones ambientales favorables de convergencias costeras que logran concentrar y retener huevos en la costa y así, evitar el intenso transporte hacia el océano. Sin embargo, los rangos de temperatura determinados en este estudio serían diferentes. Las diferencias en rangos de temperatura encontradas estarían dentro de los rangos en que se encuentran habitualmente los huevos de ambas especies a lo largo de Chile, siendo el rango de sardina común más estrecho $\left(11-12^{\circ} \mathrm{C}\right.$; Cubillos et al. 2001) que el de anchoveta (Claramunt et al. 1996, Cubillos et al. 2005).

\section{Abundancia de huevos y larvas en relación a la SALINIDAD}

Nuñez et al. (1995) en la costa de la región de Concepción $\left(36^{\circ}\right.$ a $\left.37^{\circ} 30^{\prime} S\right)$ detectaron una correlación positiva entre las densidades de huevos y larvas de anchoveta con la salinidad, tanto para el periodo reproductivo principal como secundario. Sin embargo, en nuestro estudio no se observó tal relación, lo que concuerda con lo reportado por Castro et al. (1997), Cubillos et al. (2005 y 2006) durante el periodo reproductivo principal entre la región de Valparaíso a Concepción. En la zona oceánica (> 20 $\mathrm{mn})$ y en las proximidades de la desembocadura del río Itata en los meses de agosto-septiembre (2002-2004) y noviembre (2001) se encontraron salinidades que variaron entre los 32 a 34 ups, presentando agrupaciones de huevos y larvas tanto de anchoveta como sardina común, lo que concuerda con los resultados obtenidos anteriormente para una zona más extensa (35-37º S) por Castro et al. (1997),
Cubillos et al. (2005 y 2006). Estos autores determinaron que para la época de máxima actividad reproductiva, la presencia de estadios tempranos de anchoveta y sardina común, era frecuente con valores de salinidad entre los 33,4 a 34,8 ups. Durante el periodo reproductivo secundario (marzo 2002), en el presente estudio se obtuvo un rango de salinidad más estrecho (33-34 ups) que en los meses de invierno con altas abundancias de huevos y larvas para ambas especies, cuyos valores de salinidad son concordante con los 34,00-34,49 ups reportados por Nuñez et al. (1995). Nuestros resultados, por lo tanto, señalan que el rango de salinidades más común, con mayores abundancias de ambas especies en la zona adyacente a la desembocadura del río Itata, sería entre 32 y 34 ups aunque ocasionalmente podría observarse agrupaciones a salinidades menores.

\section{Proximidad a la desembocadura del Río Itata}

Los huevos y larvas de anchoveta ocurrieron en las proximidades de la desembocadura del río Itata, aunque en concentraciones variables, prácticamente todos los años durante los periodos de mayor reproducción de esta especie (agosto-septiembre). Durante el periodo secundario de desove (marzo 2002), se cuantificó altas abundancias de larvas de anchoveta en las proximidades de la desembocadura y también más al sur sobre la plataforma continental frente a Bahia Coliumo, lo que no concuerda con Nuñez et al. (1995), quien reporta en verano (febreromarzo) altas densidades de huevos y larvas pero asociadas a la plataforma continental de la Terraza del Itata. El papel de la desembocadura como zona de concentración larval en el periodo de fines de verano (marzo) 2002, no es tan claro, por cuanto las abundancias de larvas reportadas en distintos años en esa zona han sido variables. Como el número de años analizados con data de marzo es bajo, una conclusión más robusta al respecto sólo se logrará sólo con un mayor número de muestreos en el futuro.

En el caso de la sardina común, durante el periodo de mayor desove de esta especie se cuantificó bajas abundancias de huevos y larvas prácticamente todos los años (excepto 1996). Durante los meses fuera del periodo de mayor desove (noviembre y marzo), no se presentaron huevos ni larvas de sardina común (noviembre 2001) o bien sus abundancias fueron muy bajas (marzo 2002). Nuestros resultados, sin embargo, nuevamente contrastan con los observados por Nuñez et al. (1995) durante el periodo de fines de verano ya que encontraron altas abundancias de sardina común en las estaciones cercanas a la desembocadura del río Itata. 
La información generada en el presente estudio señala que el área inmediatamente adyacente a la desembocadura del río Itata (entre 0,5 y las 4 primeras millas náuticas hacia el oeste) no sería una zona importante de agregación de huevos y larvas de anchoveta y sardina común en los periodos considerados.

Sepúlveda et al. (2000) describieron que la pluma proveniente del sector del río Itata tiene un área de influencia hasta alrededor de las $15 \mathrm{mn}$ desde la costa (periodo invernal). En nuestro estudio en los diferentes años no se observó tal estructura, excepto en septiembre del 2005 donde el área de influencia alcanzó sobre las 19 mn (costa-océano) y en sentido latitudinal abarcó hasta las $30 \mathrm{mn}$ aproximadamente, área donde encontramos la desembocadura del río Itata ( $\left.36^{\circ} 22^{\prime} S\right)$. En esta zona se observó un gradiente horizontal de salinidad (20 a 34 ups), donde se hallaron pequeñas agrupaciones de huevos y larvas de anchoveta y sardina común. La presencia de huevos en esta zona de gradientes podría ser parte de una estrategia reproductiva por parte de los adultos (i.e., posición desove), ya que se ha descrito para la zona adyacente al Itata mayores abundancias de oferta de alimento para las larvas de anchoveta y sardina común (Nuñez et al. 1995). Sin embargo, en nuestro estudio en sólo uno de los 5 inviernos estudiados se observó esta posible relación de abundancias de ictioplancton con los gradientes de salinidad por lo que la potencial importancia de éstos, como factores de agregación de huevos y larvas, sólo podría proponerse para años en que estas estructuras (gradientes de salinidad) son más marcadas (i.e., 2005 en nuestro estudio).

La ausencia de altas abundancias de huevos de ambas especies en la zona inmediatamente adyacente a la desembocadura del río Itata no significa que el aporte de agua dulce sea poco importante en el desarrollo y sobrevivencia de los primeros estadios de desarrollo de estas especies. El aporte de agua dulce del río junto a los demás ríos de la zona pueden ser muy importantes en años muy lluviosos, i.e., los ríos Itata y Bío-Bío provocan flujos geostróficos que facilitan el intercambio plataformaocéano (Sobarzo 1994, 1999), o pueden constituir un factor relevante en la zona por cuanto aumenta la estabilidad de la columna de agua en sus primeros metros. Al disminuir el efecto potencial de la turbulencia generada por el viento en superficie, consecuentemente, se podría estar contribuyendo al aumento de la sobrevivencia larval en días de intensas tormentas de vientos norte, típicos en la zona durante el invierno, estación en que el aporte de aguas del río Itata es mayor (junio a julio $\sim 600 \mathrm{~m}^{3} \mathrm{~s}^{-1}$; Sobarzo et al. 2007).

En otras especies de peces pelágicos y demersales se ha observado una asociación entre las zonas de desove y la desembocadura de ríos. En la anchoveta del norte (Engraulis mordax), por ejemplo, se ha observado reproducción en la desembocadura del río Columbia, donde las concentraciones de huevos se encuentran distribuidas sobre la pluma de agua dulce (Richardson 1981). En el golfo de Vizcaya los desoves de la anchoveta se asocian, en parte, a la desembocadura del río Garona (Motos et al. 1996), y en el Mediterráneo noroccidental, a las desembocaduras de los ríos Arno, Ródano y Ebro (García \& Palomera 1996). Estas asociaciones se deben a que las descargas fluviales enriquecidas de nutrientes por los aportes terrestres influyen en procesos biológicos, como el crecimiento, mortalidad y reclutamiento (Grimes 2001). Tales asociaciones han sido demostradas también, en el bacalao en la costa norte de Noruega (Skreslet 1976), el róbalo en el sur de Chile (Quiñones \& Montes 2001) y la anchoveta en aguas costeras del sudoeste de Taiwan (Tsai et al. 1997). En el caso de la anchoveta Engraulis ringens, cuya distribución latitudinal es muy amplia, las más altas abundancias de individuos corresponden a poblaciones ubicadas en el norte de Perú y la zona norte de Chile-sur del Perú, donde no se localizan ríos importantes. En la zona centro-sur de Chile, altas abundancias de huevos y larvas han sido descritas en la zona entre Corral y Lebu en invierno (Cubillos et al. 2005, 2006), zona que se caracteriza por un aporte importante de agua dulces provenientes tanto de la zona de Corral como de ríos menores y escorrentía a lo largo de la costa durante la época invernal de mayor desove. En esta zona las más altas abundancias de huevos y larvas de anchoveta y sardina común no se encuentran en las aguas de menor salinidad sino fuera de la pluma de agua más dulce. En nuestro estudio tampoco se observó alta abundancias de huevos ni larvas dentro de la pluma del río Itata. El área inmediatamente adyacente a la desembocadura presentó en general durante los periodos estudiados, bajas abundancias de huevos y larvas de estas especies, excepto el invierno del año 2005. Por lo tanto, la importancia del aporte de aguas dulces del río Itata, como generador de estructuras frontales acumuladoras de huevos y larvas de estas especies, en las escalas espaciales y temporales aquí analizadas, no es permanente sino que parece ser muy variable inter-anualmente. 


\section{Agradecimientos}

Estudio financiado por Programa PIMEX-Nueva Aldea. La data de ictioplancton fue generada en el marco de proyectos del Fondo de Investigación Pesquera y proyectos FONDECYT. Los autores agradecen la colaboración en cruceros y separación de muestras al personal de Laboratorio de Oceanografía Pesquera y Ecología larval (LOPEL) de la Universidad de Concepción, especialmente al Sr. Alejandro Yañez.

\section{LITERATURA CITADA}

Agenbag JJ, AJ Richardson, H Demarcq, P Fréon, S Weeks \& F A Shillington. 2003. Estimating environmental preferences of South African pelagic fish species using catch size- and remote sensing data. Progress in Oceanography 59: 275-300.

Arcos DF, SP Nuñez, L Castro \& N Navarro. 1987. Variabilidad vertical de clorofila a en un área de surgencia frente a Chile central. Investigación Pesquera, Chile 34: 47-55.

Arcos DR, S Núñez \& AAcuña. 1996. Variabilidad de pequeña escala en la zona nerítica del sistema de surgencia de Talcahuano (Chile centro-sur): Identificación y dinámica de retención larval. Gayana Oceanología 4(1): 21-58.

Ayon P. 2001. Distribución y abundancia de huevos y larvas del stock norte-centro de la anchoveta peruana en el invierno 2000. En: Crucero de evaluación de la biomasa desovante de la Anchoveta por el método de producción de huevos (mph). BICs José Olaya Balandra y SNP-2 000809, de Punta Falsa $\left(6^{\circ} \mathrm{S}\right)$ a Tambo de Mora $\left(14^{\circ} \mathrm{S}\right)$. Informe IMARPE 162: 11-21.

Bernal C, M Canales, V Cataste, A Sepúlveda, P Ruiz, $\mathbf{R}$ Alarcón, S Núñez, C Oyarzún, S Gacitúa, G Claramunt \& G Herrera. 2004. Evaluación del stock desovante de anchoveta y sardina común entre la $\mathrm{V}$ y X Regiones, año 2003. Informe Final. Proyecto FIP-IT/2003-06: 1-169. [en línea] <http://www.fip.cl/FIP/Archivos/pdf/informes/ inffinal\%202004-03.pdf>

Boyra G, L Rueda, S Coombs, S Sundby, B Adlandsvik, M Santos \& A Uriarte. 2003. Modelling the vertical distribution of eggs of anchovy (Engraulis encrasicolus) and sardine (Sardine pilchardus). Fisheries Oceanography 12(4/5): 381-395.

Bunn N, C Fox \& T Webb. 2000. A literature review of studies on fish egg mortality: Implications for the estimation of spawning stock biomass by the annual egg production method. Funded under MAFF project code MF0426: Fish egg develpoment and mortality studies in the Irish Sea. Science Series Technical Report 111: 1-37.

Castillo J, J Cordova, S Lillo, M Barbieri, J Osses \& P Barria. 1995. Estimación del reclutamiento de anchoveta en las regiones I y II. Valparaíso, Informe Final. Proyecto FIP-IT/95-02: 1-116. [en línea] <http://www.fip.cl/FIP/ Archivos/pdf/informes/IT\%2095-02.pdf>
Castro LR. 2001. Studies of the environmental conditions and larval survival of the southernmost anchoveta stock of the Hulboldt Current during winter spawning season. Global Ocean Ecosystem Dynamic (GLOBEC), Newsletter 7(2): 15-17.

Castro LR \& E Hernández. 2000. Early life survival of the anchoveta Engraulis ringens off central Chile during the 1995 and 1996 winter spawning seasons. Transactions of the American Fisheries Society 129(5): 1107-1117.

Castro LR, GR Salinas \& EH Hernández. 2000. Environmental influences on winter spawning of the anchoveta Engraulis ringens off central Chile. Marine Ecology Progress Series 197: 247-258.

Castro LR, G Claramunt, MC Krautz, A Llanos-Rivera \& P Moreno. 2009. Egg trait variation in anchoveta Engraulis ringens: a maternal response to changing environmental conditions in contrasting spawning habitats. Marine Ecology Progress Series 381: 237-248.

Castro L, R Quiñones, H Arancibia, D Figueroa, R Roa, M Sobrazo \& M Retamal. 1997. Áreas de desove de anchoveta y sardina común en la zona central. Informe Final. Proyecto FIP-IT/96-11: 1-115. [en línea] <http:// www.fip.cl/FIP/Archivos/pdf/informes/IT\%2096-11.pdf>

Claramunt G, G Herrera, P Pizarro, J Pizarro, R Escribano, M Oliva, A Olivares, C Guerra \& A Zuleta. 1996. Evaluación del reclutamiento de anchoveta en las Regiones I y II. Informe Final. Proyecto FIP 95-02: 1-81. [en línea] $<$ http://www.fip.cl/FIP/Archivos/pdf/informes/IT\%209502.pdf>

Cubillos L \& H Arancibia. 1993. On the seasonal growth of common sardine (Strangomera bentincki) and anchovy (Engraulis ringens) off Talcahuano, Chile. Revista de Biología Marina 28(1): 43-49.

Cubillos L, D Arcos, M Canales \& D Bucarey. 2001. Seasonal growth of small pelagic fish off Talcahuano $\left(37^{\circ} \mathrm{S}-73^{\circ} \mathrm{W}\right)$, Chile: a consequence of their reproductive strategy to seasonal upwelling? Aquatic Living Resources 14: 115124.

Cubillos L, D Bucarey \& M Canales. 2002. Monthly abundance estimation for common sardine Strangomera bentincki and anchovy Engraulis ringens in the centralsouthern area off Chile (34-40${ }^{\circ}$ S). Fisheries Research 57: 117-130.

Cubillos L, L Castro \& C Oyarzún. 2005. Evaluación de stock desovante de anchoveta y sardina común entre la V y X Regiones, año 2004. Informe Final. Proyecto FIP 200403: 1-130. [en línea] <http://www.fip.cl/FIP/Archivos/pdf/ informes/inffinal\%202004-03.pdf>

Cubillos L, L Castro, G Claramunt \& C Oyarzún. 2006. Evaluación de stock desovante de anchoveta y sardina común entre la V y X Regiones, año 2005. Informe Final. Proyecto FIP 2005-02: 1-147. [en línea] < http://www.fip.cl/ FIP/Archivos/pdf/informes/inffinal\%202005-03.pdf>

Dorsey S, E Houde \& J Gamble. 1996. Cohort abundances and daily variability in mortality of eggs and yolk-sac larvae 
of bay anchovy, Anchoa mitchilli, in Chesapeake Bay. Fishery Bulletin 94: 257-267.

Drinkwater KF \& KT Frank. 1994. Effects of river regulation and diversion on marine fish and invertebrates. Aquatic Conservation: Freshwater and Marine Ecosystem 4: 135151.

Escribano R, R Venegas, J Pizarro \& G Claramunt. 1996. Temperature-dependent development rate of eggs of the «anchoveta» Engraulis ringens from northern Chile. Estudios Oceanológicos 15: 39-46.

Frimodt C. 1995. Multilingual illustrated guide to the world's commercial coldwater fish, 215 pp. Fishing News Books, Osney Mead, Oxford.

Fuiman LA. 2002. Special considerations of eggs and larvae. En: Fuiman L \& R Werner (eds). Fishery science: The unique contributions of early life stages, pp. 1-31. Blackwell Science, Oxford.

García A \& I Palomera. 1996. Anchovy early life history and its relation to its surrounding environment in the Western Mediterranean basin. Scientia Marina 60(2): 155-166.

Grimes CB. 2001. Fishery production and the Mississippi River discharge. Fisheries 26(8): 17-26.

Grimes Ch \& J Finucane. 1991. Spatial distribution and abundance of larval and juvenile fish, chlorophyll and macrozooplankton around the Mississippi River discharge plume, and the role of the plume in fish recruitment. Marine Ecology Progress Series 75: 109-119.

Herrera G, E Tarifeño \& MC Orellana. 1987. Descripción de huevos y primeras fases larvales de la sardina común (Strangomera bentincki) y del machuelo (Ethmidium maculatum). Biología Pesquera 16: 107-113.

Hormazabal S, S Nuñez, D Arcos, F Espindola \& G Yuras. 2004. Mesoscale eddies and pelagic fishery off central Chile (33-40º). Gayana 68(2): 291-296.

Landaeta MF. 2007. Efecto parental en la variabilidad estacional de las características del desove de merluza común frente a Chile central. Tesis Doctoral, Departamento de Oceanografía, Universidad de Concepción, Concepción, $197 \mathrm{pp}$.

Landaeta MF \& LR Castro. 2002. Spring spawning and early nursery zone of the mesopelagic fish Maurolicus parvipinnis at the coastal upwelling zone off Talcahuano, central Chile. Marine Ecology Progress Series 226: 179191.

Llanos-Rivera A. 2005. Variabilidad en las características de los estadios tempranos de Engraulis ringens entre zonas de desoves a lo largo de Chile. Tesis Doctoral en Ciencias Biológicas, Área Zoología, Universidad de Concepción, Concepción, 154 pp.

Lloret J, I Palomera, J Salat \& I Sole. 2004. Impact of freshwater input and wind on landings of anchovy (Engraulis encrasicolus) and sardine (Sardina pilchardus) in shelf waters surrounding the Ebre (Ebro) River delta (north-western Mediterrancan). Fisheries Oceanography 13(2): 102-110.

Morales CE, HE González, SE Hormazabal, G Yuras, J Letelier \& LR Castro. 2007. The distribution of chlorophyll- $a$ and dominant planktonic components in the coastal transition zone off Concepción, central Chile, during different oceanographic conditions. Progress in Oceanography 75: 452-469.

Moser H \& E Ahlstrom. 1985. Staging anchovy eggs. En: Lasker R (ed). An egg production method for estimating spawning biomass of pelagic fish: Application to the northern anchovy, Engraulis mordax. NOAA Technical Report NMFS 36: 37-41.

Motos L, A Uriarte \& V Valencia. 1996. The spawning environment of the Bay of Biscay anchovy (Engraulis encrasicolus L.). Scientia Marina 60(Suppl. 2): 117-140.

Nuñez S, L Cubillos, D Arcos, A Urrutia, V Troncoso, F Véjar, M Landaeta, R Quiñones, A Pacheco, H Muñoz \& M Braun. 1995. Condiciones oceanográficas que inciden en el reclutamiento de los recursos anchoveta y sardina común en la VIII Región. Informe Final. Proyecto FIP-IT/ 95-11: 1-473. [en línea] <http://www.fip.cl/FIP/Archivos/ pdf/informes/IT\%2095-11.pdf>

Oliva J, H Reyes, V Varos, E Diaz, C Montenegro, V Catasti, G Claramunt, G Herrera, J Pizarro, P Pizarro \& S Soto. 2002. Evaluación del stock desovante de anchoveta por el método de producción de huevos en la I y II Regiones, año 2002. Informe Final. Proyecto FIP 2002-10: 1-89. [en línea] <http://www.fip.cl/FIP/Archivos/pdf/informes/ inffinal\%202002-10.pdf>

Parada C. 1999. Influencia de factores físicos en la variabilidad interdiaria de la distribución de estados tempranos de Engraulis ringens en el Golfo de Arauco. Tesis de Magíster en Ciencias, Mención Oceanografía, Facultad de Oceanografía, Universidad de Concepción, Concepción, $98 \mathrm{pp}$.

Parada C, M Sobarzo, D Figueroa \& L Castro. 2001. Circulación del Golfo de Arauco en un periodo de transición estacional: Un nuevo enfoque. Investigaciones Marinas 29(1): 11-24.

Parada C, C Van Der Lingen, C Mullon \& P Penven. 2003. Modelling the effect of buoyancy on the transport of anchovy (Engraulis capensis) eggs from spawning to nursery grounds in the southern Benguela: an IBM approach. Fisheries Oceanography 12(3): 170-184.

Quiñones RA \& RM Montes. 2001. Relationship between freshwater input to the coastal zone and the historical landings of the benthic/demersal fish Eleginops maclovinus in central-south, Chile. Fisheries Oceanography 10(4): 311328.

Richardson SL. 1981. Spawning biomass and early life of northern anchovy, Engraulis mordax, in the northern subpopulation off Oregon and Washington. Fishery Bulletin 78(4): 855-875. 
Rodríguez-Graña L \& L Castro. 2003. Ichthyoplankton distribution off the Peninsula de Mejillones, Chile $\left(23^{\circ} \mathrm{S}\right.$, $71^{\circ} \mathrm{W}$ ), under variable hydrographic conditions during the austral summer and winter of the 1997 El Niño. Hydrobiologia 501: 59-73.

Sepúlveda A, L Cubillos, S Nuñez, T Canales, D Bucarey, A Rojas, J Oliva, P Barria, E Diaz, V Baros \& H Miranda. 2000. Antecedentes biológicos del stock desovante de anchoveta y sardina común de la V a IX Regiones. Informe Final. Proyecto FIP-IT/97-04: 1-199. [en línea] <http:// www.fip.cl/FIP/Archivos/pdf/informes/inffinal\%209704.pdf>

Serra R. 1978. La pesquería de sardina común (Clupea Strangomera bentincki) y anchoveta (Engraulis ringens) de Talcahuano: análisis de su desarrollo y situación actual. Investigación Pesquera, Chile 29: 1-21.

Serra R. 1986. Desarrollo de la pesquería de anchoveta (Engraulis ringens) y los cambios de su abundancia. Investigación Pesquera, Chile 33: 13-24.

Serra R, O Rojas, M Aguayo, F Inostroza \& J Cañón. 1979. Sardina común. En: CORFO-IFOP (ed). Estado actual de las principales nacionales. Bases para un Desarrollo Pesquero. Peces I, 36 pp. CORFO-IFOP, Valparaíso.

Skreslet S. 1976. Influence of freshwater outflow from Norway on recruitment to the stock of Arcto-Norwegian cod. En: Skreslet S, R Leinebo, JBLMathews, E Sakshaug (eds). Freshwater on the sea, pp. 233-237. The Association of Norwegian Oceanographers, Oslo.
Sobarzo M. 1994. Oceanografía física entre Punta Nugurne

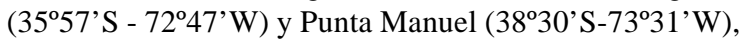
Chile: una revisión histórica (1936-1990). Gayana Oceanología 2: 5-17.

Sobarzo M. 1999. Surgencia costera sobre una plataforma continental limitada por cañones submarinos, Concepción, Chile central (3640’S;73¹5’W). Tesis de Doctorado, Facultad de Oceanografía, Universidad de Concepción, Concepción, 236 pp.

Sobarzo M, L Bravo, D Donoso, J Garcés-Vargas \& W Schneider. 2007. Coastal upwelling and seasonal cycles that influence the water column over the continental shelf off central Chile. Progress in Oceanography 75: 363-382.

Tsai CF, PY Chen, CP Chen, MA Lee, GY Shiah \& KT Lee. 1997. Fluctuation in abundance of larval anchovy and environmental conditions in coastal waters off southwestern Taiwan as associated with the El Niño-Southern Oscillation. Fisheries Oceanography 6(4): 238-249.

Valle-Levinson A, LP Atkinson, D Figueroa \& L Castro. 2003. Flow induced by upwelling winds in an equatorward facing bay; Gulf of Arauco, Chile. Journal of Geophysical Research (Oceans) 108(C2): 3054. <doi: 10.1029/ 2001JC1272, 2003>

Vargas C, G Valenzuela, S Nuñez \& D Arcos. 1996. Mortalidad natural de larvas de merluza común (Merluccius gayi gayi) frente a Chile centro-sur en relación a su distribución espacial y grado de agregación. Investigaciones Marinas 24: 19-30.

Recibido el 4 de mayo de 2010 y aceptado el 10 de septiembre de 2010 